Arch. Tierz., Dummerstorf 43 (2000) 5, 463-476 Aus dem Institut fur Tierzucht und Haustiergenetik der Universitat Gottingen' und dem Institut fur Tierzucht und
Tierverhalten der FAL Mariensee ${ }^{2}$

SIGURD LAUBE', MARTINA HENNING ${ }^{2}$, HORST BRANDT $^{1}$, ERHARD KALLWEIT ${ }^{2}$ und PETER GLODEK'

\title{
Die Fleischbeschaffenheit von Schweinekreuzungen mit besonderen Qualitätseigenschaften im Vergleich zum heutigen Standard- und Markenschweineangebot*
}

Herrn Professor Dr. Dr. h.c. Gerhard von Lengerken zum 65. Geburtstag gewidmet

\begin{abstract}
Summary
Title of the paper: Meat Quality in Pig Crosses with special quality characteristics as compared to present Standard and Brand Pork Supply

In a field experiment in Niedersachsen products of six sire lines mated to BHZP hybrid sows of one herd were compared. They were fattened in 4 BEG-farms and slaughtered at the Premium abattoir Zeven, where meat quality traits were measured on about 3000 pig carcasses (300-800 per genetic group).

It is shown that the present market standard from stress susceptible PI(PP)-boars produces $20 \%$ PSE carcasses and the present quality brand standard from $\mathrm{PI}^{*} \mathrm{HA}(\mathrm{NP})$ boars still $10 \%$, whereas all stress resistent (NN) products range below $2 \%$ PSE. Hampshire crosses cause higher water -, reduced protein contents and lower final $\mathrm{pH}$ values among their progeny because of their disturbed glucose metabolism (RN-defect gene). Only in progeny of Danish Duroc boars the intramuscular fat content reached the desired $2 \%$, German products ranged between $1.2-1.5 \%$. Recommendations for breeding more uniform endproducts with higher quality standards are given.
\end{abstract}

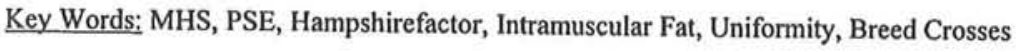

\section{Zusammenfassung}

In einem Feldversuch in Niedersachsen wurden die Endprodukte von sechs Vaterrassenkombinationen in Anpaarung an BHZP-Hybridsauen eines Ferkelerzeugerbetriebes, die in 4 BEG-Betrieben (BEG=Bauernsiegel-Erzeuger-Gemeinschaft) gemåstet und auf dem Premiumschlachthof Zeven versuchsmässig ausgeschlachtet wurden, verglichen. In dieser Arbeit sind die Fleischbeschaffenheitskriterien an etwa 3000 Versuchstieren (300-800 je Vaterrasse) analysiert worden.

Es wird gezeigt, dass das heutige Standardangebot (Nachkommen von PI(PP)-Ebern) $20 \%$ PSE-Schlachtkörper liefert und das von PI*HA(NP)-Ebern (heutiger Markenstandard) noch $10 \%$, während alle reinerbig stressresistenten Endprodukte unter $2 \%$ liegen. Hampshirekreuzungseber bewirken bei ihren Nachkommen wegen des gestörten Glucosestoffwechsels (RN-Defektgen) erhohte Wasser- und emiedrigte Proteingehalte und einen deutlich niedrigeren End-PH-Wert. Der intramuskuläre Fettgehalt erreicht nur bei Nachkommen dänischer Duroceber die erwünschten $2 \%$ und liegt bei deutschen Herkünten zwischen 1,2-1,5\%. Empfehlungen fur die Zucht qualitativ hochwertigerer und ausgeglichenerer Markenschweine werden gegeben.

Schlüsselwörter: MHS, PSE, Hampshirefaktor, Intramuskuläres Fett, Uniformităt, Rassenkreuzungen

1. Einleitung

Den Anlass für dieses vom Land Niedersachsen und Unternehmen der beteiligten Wirtschaft geförderten Forschungsvorhabens lieferten Klagen über das Angebot einer - Gefordert durch Mittel des Niedersachsischen Ministeriums fur ELF, der Premiumfleisch AG, Zeven, der Nordland EG, Uelzen und der
Zuchtungszentrale Deutsches Hybridschwein, Luneburg 
grossen bäuerlichen Markenschweineerzeugergemeinschaft aus deren Vermarkter- und Abnehmerkreis im Lebensmittelhandel. Die wichtigsten Kritikpunkte hinsichtlich der Fleischbeschaffenheit betrafen:

1) die Unausgeglichenheit in früh- und spätpostmortalen Qualitätskriterien unter praxisüblichen Schlacht- und Vermarktungsbedingungen, die zu wechselnden Frequenzen bekannter Fleischfehler (vor allem PSE-Fleisch) führe,

2) das Fehlen besonders auslobungsfähiger zusätzlicher Qualitätseigenschaften, etwa den Genuss- oder Gesundheitswert betreffend, gegenüber den zahlreichen Konkurrenzangeboten am Markt.

Als wesentliche Ursachen für diese Mängel waren zu untersuchen:

zu 1) Die Verwendung zu vieler verschiedener und häufig nicht reinerbig stressstabiler Vaterrassen in deutschen Markenprogrammen, welche zu Endprodukten mit teilweise mischerbigem MHS-Status führen. Deren Schlachtkörper bilden nach SCHMITTEN (1993) unter weniger schonenden Schlachtbedingungen vermehrt PSE aus. Ausserdem werden in Markenprogrammen heute häufig Hampshire-Kreuzungseber eingesetzt, welche das dominante $\mathrm{RN}^{-}-\mathrm{Gen}$ (den sog. Hampshirefaktor) an ihre Nachkommen vererben, das zu Wasserbindungsdefekten im Sinne eines spätpostmortal eintretenden PSE-Effektes führt (LEBRET et al., 1999).

zu 2) Das wichtigste, bisher am deutschen Markt wie in der Zucht gar nicht berücksichtigte Geschmackskriterium ist der intramuskuläre Fettgehalt (IMF), der sich bei allen deutschen Herkünften seit Jahren verringert hat und heute nur noch weniger als halb so hoch ist, wie er für Qualitätsschweinefleisch von Experten gefordert wird (HOFER und SCHWÖRER, 1996).

Diese Untersuchung, die Teil eines Gemeinschaftsprojektes von drei Forschungsinstituten ist, beschäftigt sich mit dem Vergleich der angesprochenen Fleischqualitätseigenschaften zwischen den Endprodukten von sechs hinsichtlich ihres MHS- und RNStatus sowie ihres IMF-Gehaltes unterschiedlich veranlagten Vaterrassen aus Deutschland und Dänemark. Dabei sollte geprüft werden, ob für Markenprogramme besser geeignete Endprodukte gefunden werden können, die dem Verbraucher eine höhere Genussqualität bieten, ohne bei den Erzeugern wirtschaftliche Einbussen zu verursachen.

\section{Material und Methoden}

\subsection{Versuchsplanung und Datenerhebung}

Die genaue Planung des Gesamtversuches ist bei PAULUS et al. (2000) beschrieben, hier sollen daher nur ihre für die Fleischbeschaffenheitskriterien relevanten Teile dargestellt werden. Es wurden die in Tabelle 1 aufgeführten sechs Kreuzungskombinationen in einem Ferkelerzeugerbetrieb erzeugt, auf 4 nachgeordnete Mastbetriebe verteilt, nach BEG-Regeln gemästet und anschliessend jeweils an einem Schlachttag pro Woche in die Routineschlachtung auf dem Premiumfleisch-Schlachthof Zeven eingegliedert, wo die u.g. zusätzlichen Datenerhebungen zur Fleischqualität erfolgten.

Genetisch war jede Kreuzungskombination mit 10 möglichst repräsentativ ausgewählten Besamungsebern, jeder mit 10 Anpaarungen an BHZP-Hybridsauen geplant, vertreten. Der Versuch fand in zwei Durchgängen von je 20 Wochen statt, wobei die 
PI(NN)- und PI*HA(NN)-Versuchsgruppen in beiden Durchgängen als Standard mit z.T. gleichen Ebern eingesetzt wurden. Im ersten Durchgang wurden sie mit den beiden deutschen Herkünften von PI(PP)- (nicht markenfähiges Normalangebot) und PI*HA(NP), dem derzeitigen Markenstandardprodukt, verglichen. Im zweiten Durchgang traten an deren Stelle die beiden dänischen Herkünfte mit DU(NN)- und HA*DU(NN)-Vätern, die wegen ihres hohen intramuskulären Fettgehaltes geprüft werden sollten.

Tabelle 1

Genetische Gruppen, MHS-Status, Anzahl Văter, gekennzeichnete Ferkel und Anzahl Tiere mit Fleischbeschaffenheitsmessungen (FQ-Daten) (Genetic Groups, MHS-Status, number of sires, earmarked and quality tested progeny pigs)

\begin{tabular}{lccccc}
\hline Vătergruppe & $\begin{array}{c}\text { Anzahl } \\
\text { Văter }\end{array}$ & Durchgang & $\begin{array}{c}\text { MHS-Status } \\
\text { der Endprod. }\end{array}$ & $\begin{array}{c}\text { Anzahlen } \\
\text { Ferkel }\end{array}$ & $\begin{array}{c}\text { Schweine mit FQ- } \\
\text { Daten*) von - bis }\end{array}$ \\
\hline PI(NN) & 18 & $1+2$ & NN & 966 & $478-681$ \\
PI(PP) & 10 & 1 & NP & 551 & $319-409$ \\
PI*HA(NN) & 14 & $1+2$ & NN & 1200 & $558-856$ \\
PI*HA(NP) & 10 & 1 & $1 / 2$ NN, 1/2 NP & 482 & $244-369$ \\
DU(NN) & 10 & 2 & NN & 547 & $233-359$ \\
HA*DU(NN) & 10 & 2 & NN & 657 & $271-436$ \\
\hline insgesamt & 72 & & & 4403 & $2103-3110$ \\
\hline
\end{tabular}

*) Die niedrigen Zahlen gelten fur die Glykogenbestimmung

Die Erhebung der Fleischbeschaffenheitsmerkmale erfolgte an insgesamt 36 Schlachttagen (zwischen dem 19.03 .98 und dem 21.01.99) auf dem PremiumSchlachthof Zeven an jeweils zwei aufeinanderfolgenden Tagen.

Am Schlachttag wurden die Schweine nach einer zweistündigen Ruhepause mit einer Butina $\mathrm{CO}_{2}$-Anlage in Zweiergondeln ca. 90 Sekunden lang betäubt. Unmittelbar danach wurden die Tiere an einer Hintergliedmasse aufgehängt und im Hängen mit dem Hohlmesser entblutet. Danach gelangten die Schweine in den Brühkessel und wurden nach dem Brühen in ihrer endgültigen Reihenfolge in das Schlachtband eingehängt. Die Schlachtkörper durchfuhren den Peitschenwäscher und gelangten nach dem Aufdruck der Schlachtnummer in den reinen Teil der Schlachtkette. Dort wurden das FOM-Protokoll (23 min. p.m.) mit dem Reflexionswert (RW) und der pH-Wert 25 min p.m. (pH1.1) bestimmt. Nach der Messung durchliefen die Schlachtkörper den Schocktunnel bei einer Temperatur von $-18^{\circ} \mathrm{C}$. Erst nach dem Schocktunnel wurden im Kühlhaus 85 min p.m. (1. Durchgang), bzw. 145 min p.m. (2. Durchgang) der zweite $\mathrm{pH}$-Wert ( $\mathrm{pH}$ 1.2) und der erste Leitfähigkeitswert (LF1 im Schinken) ermittelt. Die Messung des pH-Wertes erfolgte zwischen der 13. und 14. Rippe im M. longissimus dorsi der jeweils rechten Schlachtkörperhälfte. Die unterschiedlichen pH1.2- und LF1-Messzeiten sind durch eine Verlängerung der Passagedauer im Schocktunnel aufgrund von Umbaumassnahmen entstanden.

Am Zerlegetag wurden die Hälften sortiert, wobei die linken Hälften in die Zerlegung gelangten und an den rechten Hälften 24 h p.m. die Leitfähigkeit (LF24) und der pHWert (pH24) mittels Doppelmessung zwischen der 13. und 14. Rippe im M. longissimus dorsi gemessen wurden. Danach wurden die Hälften zwischen der 13. und 14.Rippe angeschnitten und ein Kotelettfoto gemacht. Auf der Kotelettfläche wurde eine Farbmessung $\left(L^{*} a^{*} b\right)$ durchgefürt. Hiernach wurde eine Kotelettprobe (zwi- 
schen 14. und 15. Rippe) aus dem M. longissimus dorsi entnommen und im Schlachthof auf Eis gekühlt. Die Kotelettproben wurden in Kühlbehältern in das Labor nach Mariensee gebracht und dort sofort bei $-18^{\circ} \mathrm{C}$ eingefroren. Die Messungen der $\mathrm{pH}-$ Werte wurden mit dem Knick pH-Meter, die Leitfähigkeitsmessungen mit dem LFStar (Matthäus) durchgeführt. Der Reflexionswert konnte dem FOM-Protokoll entnommen werden. Die Farbmessungen auf der Kotelettoberfläche erfolgten mit dem Chroma-Meter (Minolta).

Die prozentualen Anteile an Protein, Wasser und IMF wurden durch Nah-Infra-RotTransmissionsmessung (NIT; Infratec 1255), der Gehalt an Glykogen durch photometrische Messungen nach Extraktherstellung ermittelt. Die Kalibrierkurve für das NITGerät wurde anhand einer nasschemischen Analyse (Extraktion mit vorgeschalteter Hydrolyse; § 35 LMBG) und der parallel durchgeführten Spektralanalyse für 120 Proben erstellt. Die Regressiongleichung konnte durch einen unabhängigen Probensatz validiert werden.

\subsection{Rohdaten und Auswertungsmethoden}

Die üblichen statistischen Parameter an den Rohdaten sind in Tabelle 2 dargestellt.

Tabelle 2

Anzahl der Beobachtungen, Rohmittelwert, Standardabweichung, Minimal- und Maximalwert der untersuchten Qualitătskriterien (Number of observations, Mean, Standard Deviation and Extremes for analyzed quality traits)

\begin{tabular}{lrrrrr}
\hline \multicolumn{1}{c}{ Messwert } & $\mathrm{N}$ & Mittelwert & Std.-abw. & Minimum & Maximum \\
\hline pH1.1 & 3068 & 6,41 & 0,21 & 5,55 & 7,12 \\
pH1.2 (85 min) & 1573 & 6,10 & 0,34 & 5,20 & 7,19 \\
pH1.2 (145 min) & 1427 & 6,22 & 0,27 & 5,23 & 6,99 \\
RW & 3139 & 24,33 & 4,22 & 14,00 & 58,00 \\
LF1 (Schinken) & 2906 & 3,03 & 0,88 & 1,00 & 9,90 \\
pH24 & 2962 & 5,51 & 0,14 & 5,15 & 6,35 \\
LF24 & 2981 & 3,39 & 1,03 & 1,42 & 9,74 \\
L $^{*}$ & 2632 & 47,24 & 2,78 & 38,45 & 56,41 \\
a $^{*}$ & 2632 & 8,07 & 1,05 & 4,69 & 12,47 \\
b $^{*}$ & 2629 & 2,20 & 1,51 & $-3,33$ & 7,11 \\
Wasser \% & 2706 & 74,96 & 0,68 & 68,44 & 78,45 \\
IMF \% & 2706 & 1,49 & 0,60 & 0,41 & 10,82 \\
Protein \% & 2706 & 23,22 & 0,91 & 19,96 & 26,41 \\
Glykogen 1* & 1073 & 12,68 & 16,71 & 0,05 & 71,69 \\
Glykogen 2** & 1030 & 1,35 & 1,52 & 0,05 & $20,79^{\text {*** }}$ \\
\hline
\end{tabular}

- Glykogen $1(\mu \mathrm{mol} / \mathrm{g})=$ Văter mit Hampshireanteil: $\mathrm{PI}^{\circ} \mathrm{HA}(\mathrm{NN}), \mathrm{PI}^{\circ} \mathrm{HA}(\mathrm{NP}), \mathrm{HA}{ }^{\circ} \mathrm{DU}(\mathrm{NN})$

* Glykogen $2(\mu \mathrm{mol} / \mathrm{g})=$ Vater ohne Hamphireanteil: PI(NN), PI(PP), DU(NN)

innerhalb dieser Glykogengruppe wurden nur drei Proben mit ober $10,0 \mu \mathrm{mol} / \mathrm{g}$ Glykogen gefunden.

Weil der pH1.2 im ersten Durchgang 85 min p.m. und im zweiten Durchgang 145 min p.m. gemessen worden ist, werden die Werte gesondert aufgeführt. Dass die im 2. Durchgang eine Stunde später gemessenen pH1.2-Werte höher waren als die früher gemessenen im 1. Durchgang ist dadurch begründet, dass im 2. Durchgang nur MHS(NN)-Tiere erfasst wurden, während sich im 1. Durchgang PI(PP)- und PI*HA(NP)-Nachkommen mit hohen pH-Werten befanden. Die im Labor ermittelten Glykogenwerte der Endprodukte unterscheiden sich erheblich zwischen den Vaterlinien, so dass eine Unterteilung der Vaterlinien in eine Gruppe mit Hampshire-(Glykogen1) und eine ohne Hampshireanteil (Glykogen2) möglich ist. 
Vor der statistischen Auswertung wurden die auf dem Schlachthof erhobenen Messwerte schlachttagskorrigiert. Die Schlachttagskorrektur wurde anhand der Schlachttagsmittel über die Nachkommen der drei an jedem Schlachttag vorhandenen Vaterlinien (PI(NN), PI*HA(NN) und einer Betriebskontrollgruppe von PI*HA(NP)-Natursprungebern, die zu diesem Zweck über den gesamten Versuch mitgeführt wurde) berechnet. Für die weiterführende Auswertung wurden nur die sechs Versuchsgruppen berücksichtigt. Der Einfluss der fixen Effekte wurde mit Hilfe der Prozedur GLM (SAS, 1996) geschätzt, wobei der Varianzanalyse folgendes Modell zugrunde lag:

$$
\begin{aligned}
& Y_{i j k l}=\mu+V l_{i}+\operatorname{Sex}_{j}+M b_{k}+\left(V l^{*} S e x\right)_{i j}+\left(V l^{*} M b\right)_{i k}+\left(S e x^{*} M b\right)_{i k}+e_{i j k l} \\
& \mathrm{Y}_{\mathrm{ijkl}}=\text { Beobachtungswert des 1-ten Tieres } \\
& \mu \quad=\text { Gesamtmittel } \\
& \mathrm{Vl}_{\mathrm{i}} \quad=\quad \text { fixer Effekt der } \mathrm{i} \text {-ten Vaterlinie }(\mathrm{i}=1-6) \\
& \operatorname{Sex}_{\mathrm{j}} \quad=\quad \text { fixer Effekt des } \mathrm{j} \text {-ten Geschlechtes }(\mathrm{j}=1,2) \\
& \mathrm{Mb}_{\mathrm{k}} \quad=\quad \text { fixer Effekt des k-ten Mastbetriebes }(\mathrm{k}=1-4) \\
& \left(\mathrm{Vl}^{*} \mathrm{Sex}\right)_{\mathrm{ij}}=\text { Interaktionseffekt zwischen i-ter Vaterlinie und j-tem Geschlecht } \\
& (\mathrm{Vl} * \mathrm{Mb})_{\mathrm{ik}}=\text { Interaktionseffekt zwischen i-ter Vaterlinie und k-tem Mastbetrieb } \\
& (\mathrm{Sex} * \mathrm{Mb})_{\mathrm{ik}}=\text { Interaktionseffekt zwischen j-tem Geschlecht und k-tem Mastbetrieb } \\
& \mathrm{e}_{\mathrm{ijkl}} \quad=\quad \text { zufälliger Restfehler. }
\end{aligned}
$$

Das Versuchsmanagement mit nur 2 pro Woche eingesetzten Ebern (je einer aus zwei verschiedenen Vaterlinien) gestattete nicht die Aufnahme des Ebers in das Modell. Dies bedeutet ein bestimmtes confounding mit Effekten des Mastbetriebes und kann

\begin{tabular}{|c|c|c|c|c|c|c|}
\hline & \multicolumn{6}{|c|}{ Effekte } \\
\hline & Vaterlinie & Geschlecht & Mastbetrieb & $V I^{*} \operatorname{Sex}$ & $\mathrm{VI} * \mathrm{Mb}$ & Sex*Mb \\
\hline $\mathrm{pH} 1 . \mathrm{l}$ & क* & * * & n.s. & n.s. & के & $\mathrm{n} . \mathrm{s}$ \\
\hline $\mathrm{pH} 1.2$ & *\#* & n.s. & 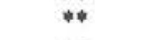 & n.s. & * & n.s. \\
\hline RW & *\#* & $* \omega *$ & n.s. & n.s. & $* * *$ & n.s. \\
\hline LF1 & 中ष & n.s. & n.s. & n.s. & - & n.s. \\
\hline $\mathrm{pH} 24$ & *** & n.s. & *\# & ** & $\% * *$ & n.s. \\
\hline LF24 & *** & n.s. & n.s. & n.s. & ** & n,s. \\
\hline $\mathrm{L}^{*}$ & $* * *$ & *** & n.s. & $* *$ & **** & n.s. \\
\hline$a^{*}$ & $* * *$ & - & $* * *$ & n.s. & \#\# & n.s. \\
\hline$b^{*}$ & $* * *$ & $\cdot$ & *** & $*$ & - & n.s. \\
\hline Wasser \% & $* * *$ & - & $* * *$ & n.s. & $* * *$ & n.s. \\
\hline IMF $\%$ & 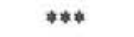 & *4* & $* *$ & $*$ & फे & ** \\
\hline Protein \% & $+\%$ & \$4* & 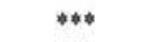 & n.s & *** & * \\
\hline Glykogen $(\mu \mathrm{mol} / \mathrm{g})$ & $* * *$ & n.s. & \#* & n.s. & $* * *$ & n.s. \\
\hline
\end{tabular}
auch zu Interaktionen Vaterlinie * Mastbetrieb führen.

3. Ergebnisse

3.1. Signifikanz der Effekte

Die folgende Tabelle 3 zeigt die im F-Test berechneten Signifikanzen für die fixen Effekte und Interaktionen.

Tabelle 3

Signifikanzen der fixen Effekte und Interaktionseffekte im F-Test (Significances of fixed and interaction effects in F-tests) 
Für sämtliche auf dem Schlachthof erhobenen Messwerte der Fleischbeschaffenheit sowie für die im Labor ermittelten Gehaltsmesswerte zeigt sich ein hochsignifikanter Einfluss der Vaterlinie. Das Geschlecht ist für den Reflexionswert, den Minolta L, den IMF- und Proteingehalt hoch, für den pH1.1-Wert mittel und für den Wassergehalt niedrig signifikant.

Der Mastbetrieb hat einen hochsignifikanten Einfluss auf den pH24, den Minolta aund b-Wert sowie auf den Wasser-, IMF- und Proteingehalt, einen mittleren auf den pH1.2 und den Glykogengehalt.

Der Interaktionseffekt zwischen Vaterlinie und Mastbetrieb (VI*Mb) zeigt für alle Messwerte einen geringen bis hochsignifikanten Einfluss, der z.T. auf die einzelnen Eber innerhalb der Vaterlinien zurückgehen dürfte.

Die beiden ubrigen Interaktionseffekte sind nur in seltenen Ausnahmefällen signifikant und daher zu vernachlăssigen.

Im folgenden soll nur auf die für das Thema relevanten Effekte der Vaterlinien, die für die Qualität ihrer Endproduktnachkommen massgeblich sind, eingegangen werden.

\subsection{Einfluss der Vaterlinie auf fruhpostmortale Fleischbeschaffenheitskriterien}

In Tabelle 4 sind die LS-Mittelwerte und Standardfehler fur die durch die Vaterlinie bestimmten Rassenkombinationen angegeben. Da vor allem der MHS-Status der Endprodukte auf diese Mittelwerte wirkt, werden im unteren Teil die Mittel der PI(PP)und der PI*HA(NP)-Gruppen dem Gesamtmittel der vier Versuchsgruppen mit ausschliesslich NN-Nachkommen (d.h. reinerbig stressstabilen Vaterrassen) gegenüber gestellt und die absoluten Differenzen in Prozent der Reststandardabweichung angegeben.

Tabelle 4

LS-Mittelwerte und Standardfehler nach Vaterlinien fur fruhpostmortale Fleischbeschaffenheitskriterien (LSMeans and Standard Errors for genetic groups in quality traits early post mortem)

\begin{tabular}{|c|c|c|c|c|}
\hline \multirow[b]{2}{*}{ Vaterlinie } & \multicolumn{4}{|c|}{ Messwert } \\
\hline & $\mathrm{pH} 1.1$ & $\mathrm{pH} 1.2$ & RW & LF1 \\
\hline $\mathrm{PI}(\mathrm{NN})$ & $6,40(0,01)$ & $6,18(0,01)$ & $23,82(0,12)$ & $3,06(0,03)$ \\
\hline PI(PP) & $6,20(0,01)$ & $5,76(0,02)$ & $22,84(0,18)$ & $3,63(0,05)$ \\
\hline $\mathrm{PI}^{\star} \mathrm{HA}(\mathrm{NN})$ & $6,42(0,01)$ & $6,19(0,01)$ & $24,42(0,11)$ & $2,98(0,03)$ \\
\hline $\mathrm{PI}^{*} \mathrm{HA}(\mathrm{NP})$ & $6,38(0,01)$ & $5,97(0,02)$ & $24,36(0,22)$ & $3,15(0,06)$ \\
\hline DU(NN) & $6,39(0,01)$ & $6,26(0,01)$ & $26,74(0,16)$ & $3,12(0,04)$ \\
\hline $\mathrm{HA}^{*} \mathrm{DU}(\mathrm{NN})$ & $6,43(0,01)$ & $6,26(0,01)$ & $26,46(0,15)$ & $2,99(0,04)$ \\
\hline Gesamtmittel & 6,38 & 6,12 & 24,58 & 3,11 \\
\hline Reststand.abw. (SE) & 0,19 & 0,26 & 2,97 & 0,80 \\
\hline $\mathrm{PI}(\mathrm{PP})-\mathrm{x}(\mathrm{NN})$ & $-0,21$ & $-0,46$ & $-2,52$ & 0,59 \\
\hline in $\mathrm{SE} \%$ & 111 & 177. & 85 & 74 \\
\hline $\left.\mathrm{PI}{ }^{*} \mathrm{HA} / \mathrm{NP}\right)-\mathrm{x}(\mathrm{NN})$ & $-0,03$ & $-0,25$ & $-0,73$ & 0,11 \\
\hline in $\mathrm{SE} \%$ & 16 & 97 & 25 & 14 \\
\hline
\end{tabular}

Am deutlichsten sind die Unterschiede zwischen Kreuzungsgruppen verschiedenen MHS-Genotyps im pH1.2, wo sie 1 - 1,8 Standardabweichungen zuungunsten des heutigen Markenstandards bzw. der PI(PP)-Nachkommen betragen. Besonders wenig diskriminativ für Nachkommen von PI*HA(NP)-Ebern sind die anderen drei Merkmale, weil sie offenbar zu fruh erhoben werden. 


\subsection{Einfluss der Vaterlinie auf spätpostmortale Fleischbeschaffenheits- kriterien}

In Tabelle 5 sind die LS-Mittelwerte für die 24 Stunden p.m. erhobenen pH- und LFMessungen wie die drei Minolta-Farbwerte zusammengestellt, auch hier wurden die summarischen Vergleiche zwischen den MHS-Genotypgruppen angefügt. Von diesen Merkmalen führt der LF24 zur deutlichsten MHS-Diskrimierung, während der pH24 eher zur Identifizierung von Hampshireanteilen geeignet erscheint. Die Minoltamessungen werden in einer gesonderten Auswertung näher analysiert.

Tabelle 5

LS-Mittelwerte und Standardfehler nach Vaterlinien fur spätpostmortale Fleischbeschaffenheitskriterien (LSMeans and Standard Errors for genetic groups in quality traits $24 \mathrm{~h}$ post mortem)

\begin{tabular}{|c|c|c|c|c|c|}
\hline \multirow[b]{2}{*}{ Vaterlinie } & \multicolumn{5}{|c|}{ Messwert } \\
\hline & $\mathrm{pH} 24$ & LF24 & Minolta L & Minolta a & Minolta b \\
\hline $\mathrm{PI}(\mathrm{NN})$ & $5,51(0,01)$ & $3,37(0,04)$ & $47,43(0,12)$ & $7,88(0,05)$ & $2,26(0,04)$ \\
\hline $\mathrm{PI}(\mathrm{PP})$ & $5,49(0,01)$ & $4.49(0,05)$ & $47,15(0,16)$ & $7,84(0,06)$ & $2,22(0,06)$ \\
\hline $\mathrm{PI}^{*} \mathrm{HA}(\mathrm{NN})$ & $5,49(0,01)$ & $3,31(0,04)$ & $47,02(0,10)$ & $8,12(0,04)$ & $2,15(0,04$ \\
\hline $\mathrm{PI}{ }^{\circ} \mathrm{HA}(\mathrm{NP})$ & $5,48(0,01)$ & $4,00(0,07)$ & $47,20(0,20)$ & $8,33(0,08)$ & $2,31(0,08)$ \\
\hline DU(NN) & $5,59(0,01)$ & $3,52(0,05)$ & $46,20(0,15)$ & $7,99(0,06)$ & $1,95(0,06)$ \\
\hline $\mathrm{HA}{ }^{*} \mathrm{DU}(\mathrm{NN})$ & $5,52(0,01)$ & $3,48(0,05)$ & $46,26(0,14)$ & $8,09(0,06)$ & $1,97(0,05)$ \\
\hline Gesamtmittel & 5,51 & 3,62 & 46,92 & 8,07 & 2,17 \\
\hline Reststand.abw. (SE) & 0,12 & 0,92 & 2,52 & 0,99 & 0,95 \\
\hline $\mathrm{PI}(\mathrm{PP})-\mathrm{x}(\mathrm{NN})$ & $-0,04$ & 1,07 & $-0,42$ & $-0,18$ & 0,14 \\
\hline in SE \% & 33 & 116 & 17 & 18 & 15 \\
\hline $\mathrm{PI} H A(N P)-x(N N)$ & $-0,05$ & 0,58 & 0,47 & $0,3 i$ & 0,23 \\
\hline in SE \% & 42 & 63 & 19 & 31 & 24 \\
\hline
\end{tabular}

\subsection{PSE- und DFD-Frequenzen in verschiedenen Kreuzungskombinationen}

Da der ublicherweise fur die PSE-Einstufung benutzte pH-Wert 45 min p.m. unter den gegebenen Schlachthofbedingungen nicht erhoben werden konnte, wurde hier empirisch eine PSE-Frequenz aus dem Mittel der in Tabelle 6 gegebenen drei Grenzkriterien bestimmt.

Tabelle 6

Empirische PSE-Bestimmung anhand des Mittels von drei Merkmalsgrenzwerten in Prozent (Empiric PSE definition as mean frequency over three quality trait limits in per cent)

\begin{tabular}{lcccc}
\hline Vaterlinie & $\mathrm{pHI}, 1<6,0$ & $\mathrm{pH} 1.2<5,6$ & LF24 $>5,5$ & Mittel \\
\hline PI(NN) & 2,20 & 1,98 & 1,97 & 2,1 \\
PI(PP) & 21,92 & 26,00 & 17,53 & 21,8 \\
PI*HA(NN) & 1,31 & 1,46 & 1,27 & 1,4 \\
PI*HA(NP) & 3,52 & 14,96 & 12,09 & 10,2 \\
DU(NN) & 2,56 & 1,45 & 1,76 & 1,9 \\
HA*DU(NN) & 0,95 & 1,21 & 2,35 & 1,5 \\
\hline
\end{tabular}

Es zeigt sich, dass der pH1.1-Grenzwert bei den PI*HA(NP)-Nachkommen nicht in das Bild der beiden anderen, besser diskriminierenden Kriterien passt, wohl aber bei den Nachkommen von NN- und PP-Vätern. Die hier gewählte PSE-Grenze ist auch nach internationalen Standards als streng einzuordnen.

Ähnlich schwierig war die in Tabelle 7 dargestellte Festlegung einer DFD-Grenze, wobei besonders die DU-Nachkommen wegen ihres deutlich höheren pH24-Wertes herausragen, so dass vor allem ihretwegen ein Grenzwert von pH24 > 5,9 vorgeschlagen wird, der ohne sie auch bei > 5,8 liegen könnte. 
LAUBE u.a.: Fleischbeschaffenheit von Schweinekreuzungen im Vergleich zum heutigen Markenschweineangebot

Tabelle 7

DFD-Frequenzen bei unterschiedlichen pH24-Grenzwerten in Prozent (DFD-Frequencies at three PH24 value limits in per cent)

\begin{tabular}{lrcc}
\hline \multicolumn{1}{c}{ Vaterlinie } & $\mathrm{pH} 24>5,8$ & $\mathrm{pH} 24>5,9$ & $\mathrm{pH}>6,0$ \\
\hline PI(NN) & 2,00 & 0,77 & 0,15 \\
PI(PP) & 0,74 & 0,25 & 0,00 \\
PI*HA(NN) & 2,30 & 1,15 & 0,38 \\
PI*HA(NP) & 2,51 & 1,11 & 0,84 \\
DU(NN) & 7,62 & 2,93 & 1,76 \\
HA $A^{*}$ DU(NN) & 2,35 & 0,94 & 0,23 \\
\hline
\end{tabular}

\subsection{Gehaltsmesswerte des Fleisches in verschiedenen Kreuzungskombina-} tionen

Die im Labor des FAL-Institutes Mariensee ermittelten Gehaltswerte sind in Tabelle 8 nach Vaterlinien dargestellt. Im unteren Teil ist die Differenz des Mittels aller Hampshirekreuzungen zu den restlichen aufgeführt, die im Glykogen-, Wasser- und Proteingehalt erheblich sind.

Tabelle 8

LS-Mittelwerte und Standardfehler für Gehaltsmesswerte nach Vaterlinien (LS-Means and Standard Errors for genetic groups in water-, protein, fat and glycogen content)

\begin{tabular}{lcccc}
\hline & & & Messwert & \\
Vaterlinie & Wasser (\%) & Protein $(\%)$ & IMF $(\%)$ & Glykogen $(\mu \mathrm{mol} / \mathrm{g})$ \\
\hline PI(NN) & $74,81(0,03)$ & $23,56(0,03)$ & $1,34(0,02)$ & $1,07(0,55)$ \\
PI(PP) & $74,97(0,04)$ & $23,61(0,05)$ & $1,23(0,03)$ & $1,82(0,74)$ \\
PI*HA(NN) & $75,09(0,02)$ & $23,00(0,03)$ & $1,47(0,02)$ & $9,93(0,52)$ \\
PI*HA(NP) & $75,48(0,05)$ & $22,60(0,06)$ & $1,37(0,05)$ & $17,58(1,08)$ \\
DU(NN) & $74,47(0,04)$ & $23,37(0,05)$ & $2,03(0,04)$ & $1,10(0,82)$ \\
HA*DU(NN) & $75,15(0,03)$ & $22,70(0,04)$ & $1,75(0,03)$ & $11,94(0,76)$ \\
\hline Gesamtmittel & 74,96 & 23,25 & 1,49 & 7,13 \\
Reststand.abw. (SE) & 0,61 & 0,80 & 0,51 & 11,57 \\
\hline x HA - x Rest & 0,49 & $-0,75$ & 0 & 11,82 \\
in SE \% & 80 & 93 & 0 & 102 \\
\hline
\end{tabular}

Hinsichtlich des intramuskulären Fettgehaltes erreichen lediglich die DU-Nachkommen im Mittel die $2 \%$-Grenze, gefolgt von den (HA*DU)-Nachkommen mit 1,75\%. Alle deutschen Kreuzungskombinationen liegen deutlich darunter, am niedrigsten mit $1,23 \%$ die von PI(PP)-Vätern.

Als Auswirkungen des sog. Hampshireeffektes zeigen alle drei diese Rasse enthaltenden Kreuzungskombinationen etwa um jeweils eine Standardeinheit höhere Glykogenund Wassergehalte sowie entsprechend niedrigere Proteingehalte als die Kombinationen ohne Hampshireanteil. Da nur die Hälfte der Nachkommen von Hampshirekreuzungsebern das dominante $\mathrm{RN}^{*}$-Schadgen erhalten, wenn es in der Hampshirerasse homozygot ist, erwartet man für sie eine deutlich zweigipflige Verteilung. Da ein $\mathrm{RN}^{-}$ -Gentest noch nicht vorhanden war, wurden die Nachkommen der drei Hampshirekreuzungen nach ihrem Glykogengehalt in die beiden Klassen $>10 \mu \mathrm{mol} / \mathrm{g}$ Fleisch (die als Träger des $\mathrm{RN}^{*}$-Gens angesehen werden) und darunter eingeteilt und für beide Klassen die Mittelwerte sowie die Differenz zwischen diesen absolut und in Standardeinheiten in Tabelle 9 zusammengestellt.

Die LS-Mittelwerte zeigen im Protein- und Wassergehalt sehr hohe Unterschiede und auch im pH24-Wert etwa 0,8 Standardeinheiten. Dagegen scheint das $\mathrm{RN}^{*}$-Gen mit 
Tabelle 9

LS-Mittelwerte fur Glykogengruppen innerhalb Hampshirekreuzungen und die Differenz zwischen ihnen in den Indikatormerkmalen (LS-Means of glycogen classes in Hampshire crosses and their difference in $\mathrm{PH} 24$ and water-, protein and fat content)

\begin{tabular}{|c|c|c|c|c|}
\hline \multirow[b]{2}{*}{ Glykogengruppe } & \multicolumn{4}{|c|}{ Messwert } \\
\hline & $\mathrm{pH} 24$ & Wasser (\%) & Protein (\%) & IMF (\%) \\
\hline$>10,0(\mathrm{I})$ & 5,43 & 75,85 & 21,80 & 1,52 \\
\hline$<10,0$ (II) & 5,52 & 75,00 & 23,16 & 1,50 \\
\hline Mittel & 5,49 & 75,18 & 22,88 & 1,51 \\
\hline Reststand.abw. (SE) & 0,11 & 0,59 & 0,74 & 0,54 \\
\hline I- II & $-0,09$ & 0,85 & $-1,36$ & 0,02 \\
\hline in $\mathrm{SE} \%$ & 82 & 144 & 184 & 4 \\
\hline Signifikanz & $\% *$ & 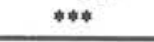 & *** & n.s. \\
\hline
\end{tabular}

dem intramuskulären Fettgehalt nicht in Beziehung zu stehen.

Hinsichtlich der Verbreitung des $\mathrm{RN}^{*}$-Gens in den deutschen und dänischen HA-Reinzuchtpopulationen gibt die Tabelle 10 Aufschluss, in der alle verwendeten HA-Kreuzungseber mit mehr als 10 geprüften Nachkommen anhand der Glykogenklassen

Tabelle 10

Glykogenklassen unter den Nachkommen von HA-Kreuzungsebern und daraus abgeleitete Frequenz von RN"Trägertieren (Glycogen and RN-Genotypes within Hampshire crossbred boars with more than 10 progenies tested)

\begin{tabular}{|c|c|c|c|c|c|c|}
\hline \multirow[b]{2}{*}{ Vaterlinie } & \multirow[b]{2}{*}{ Eber } & \multicolumn{3}{|c|}{ absolut } & \multicolumn{2}{|c|}{$\%$} \\
\hline & & $\mathrm{N}$ & $<10,0$ & $>10,0$ & $\mathrm{~m}^{+} / \mathrm{rn}^{+}$ & RN\% \\
\hline & 304 & 81 & 81 & 0 & 100 & 0 \\
\hline & 315 & 36 & 24 & 12 & 67 & 33 \\
\hline & 316 & 67 & 33 & 34 & 49 & 51 \\
\hline & 323 & 65 & 65 & 0 & 100 & 0 \\
\hline & 324 & 53 & 20 & 33 & 38 & 62 \\
\hline $\mathrm{PI}^{*} \mathrm{HA}$ & 325 & 36 & 18 & 18 & 50 & 50 \\
\hline \multirow[t]{10}{*}{$(\mathrm{NN})$} & 662 & 59 & 29 & 30 & 49 & 51 \\
\hline & 683 & 14 & 6 & 8 & 43 & 57 \\
\hline & 684 & 41 & 17 & 24 & 41 & 59 \\
\hline & 876 & 10 & 4 & 6 & 40 & 60 \\
\hline & 957 & 76 & 76 & 0 & 100 & 0 \\
\hline & 5128 & 18 & 10 & 8 & 56 & 44 \\
\hline & insgesamt & 556 & 383 & 173 & 69 & 31 \\
\hline & 303 & 23 & 12 & 11 & 52 & 48 \\
\hline & 649 & 27 & 14 & 13 & 52 & 48 \\
\hline & 650 & 35 & 16 & 19 & 46 & 54 \\
\hline $\mathrm{PI}^{*} \mathrm{HA}$ & 652 & 39 & 24 & 15 & 62 & 38 \\
\hline \multirow[t]{9}{*}{ (NP) } & 669 & 51 & 30 & 21 & 59 & 41 \\
\hline & 671 & 13 & 4 & 9 & 31 & 69 \\
\hline & 672 & 37 & 13 & 24 & 35 & 65 \\
\hline & 696 & 10 & 6 & 4 & 60 & 40 \\
\hline & insgesamt & 235 & 119 & 116 & 51 & 49 \\
\hline & 8856 & 50 & 22 & 28 & 44 & 56 \\
\hline & 8916 & 21 & 14 & 7 & 67 & 33 \\
\hline & 8912 & 9 & 3 & 6 & 33 & 67 \\
\hline & 8925 & 43 & 43 & 0 & 100 & 0 \\
\hline$H A^{\star} D U$ & 8929 & 42 & 22 & 20 & 52 & 48 \\
\hline \multirow[t]{4}{*}{$(\mathrm{NN})$} & 8935 & 26 & 12 & 14 & 46 & 54 \\
\hline & 8941 & 38 & 28 & 10 & 74 & 26 \\
\hline & 8963 & 39 & 21 & 18 & 54 & 46 \\
\hline & insgesamt & 268 & 165 & 103 & 62 & 38 \\
\hline
\end{tabular}


und des daraus abgeleiteten Anteils an $\mathrm{RN}^{*}$-Trägern aufgefulhrt sind.

Es zeigt sich, dass drei deutsche und ein dänischer NN-Eber keine RN-Nachkommen gebracht haben, offensichtlich also dieses Gen nicht besaßen. Dies bedeutet, dass in den großelterlichen HA-Populationen das $\mathrm{RN}^{-}$-Schadgen nicht fixiert ist, sondern mindestens einzelne heterozygote Tiere existieren, die Ausgangspunkt furr eine Sanierung der HA-Rasse von diesem Schadgen sein könnten.

\subsection{Ausgeglichenheit der Kreuzungskombinationen}

In Tabelle 11 wird die Streubreite (SB) für wichtige Merkmale der Fleischbeschaffenheit, berechnet als Differenz zwischen dem höchsten (max.) und dem niedrigsten (min.) Ebernachkommenmittel innerhalb Vaterlinie, beschrieben.

Tabelle 11

Streubreite (SB) zwischen höchstem und niedrigsten Nachkommenmittel der Eber innerhalb Vaterlinien (Range (SB) between highest and lowest progeny mean of boars within sire lines in meat quality traits)

\begin{tabular}{|c|c|c|c|c|c|c|c|}
\hline \multirow[b]{2}{*}{ Messwert } & \multicolumn{7}{|c|}{ Vaterlinie } \\
\hline & & $\mathrm{PI}(\mathrm{NN})$ & $\mathrm{PI}(\mathrm{PP})$ & $\mathrm{PI}{ }^{*} \mathrm{HA}(\mathrm{NN})$ & $\mathrm{PI}^{*} \mathrm{HA}(\mathrm{NP})$ & DU(NN) & $\mathrm{HA}^{*} \mathrm{DU}(\mathrm{NN})$ \\
\hline \multirow{3}{*}{ pHI.1 } & $\max$. & 6,48 & 6,31 & 6,51 & 6,45 & 6,48 & 6,50 \\
\hline & $\min$. & 6,34 & 6,03 & 6,39 & 6,29 & 6,32 & 6,38 \\
\hline & SB & 0,14 & 0,28 & 0,12 & 0,16 & 0,16 & 0,12 \\
\hline \multirow{3}{*}{$\mathrm{pH} 1.2$} & $\max$. & 6,27 & 5,87 & 6,31 & 6,12 & 6,39 & 6,41 \\
\hline & $\min$. & 6,02 & 5,70 & 6,10 & 5,80 & 6,10 & 6,16 \\
\hline & SB & 0,25 & 0,17 & 0,21 & 0,32 & 0,29 & 0,25 \\
\hline \multirow{3}{*}{ LF24 } & $\max$ & 3,82 & 5,47 & 3,53 & 4,37 & 4,07 & 3,92 \\
\hline & $\min$. & 3,06 & 3,95 & 3,10 & 3,56 & 3,09 & 3,30 \\
\hline & SB & 0,76 & 1,52 & 0,43 & 0,81 & 0,98 & 0,62 \\
\hline \multirow{3}{*}{ Protein \% } & $\max$. & 23,98 & 24,01 & 23,32 & 23,04 & 24,00 & 23,69 \\
\hline & $\min$. & 23,33 & 22,99 & 22,06 & 21,76 & 23,20 & 22,01 \\
\hline & SB & 0,65 & 1,02 & 1,26 & 1,28 & 0,80 & 1,68 \\
\hline \multirow{3}{*}{ Wasser $\%$} & $\max$. & 75,06 & 75,26 & 75,78 & 75,96 & 74,65 & 75,62 \\
\hline & $\min$. & 74,35 & 74,52 & 74,63 & 75,32 & 74,10 & 74,65 \\
\hline & SB & 0,71 & 0,75 & 1,15 & 0,64 & 0,55 & 0,97 \\
\hline \multirow{3}{*}{$\mathrm{IMF} \%$} & $\max$. & 1,71 & 1,61 & 1,73 & 1,53 & 2,57 & 1,98 \\
\hline & $\min$. & 1,09 & 0,73 & 1,14 & 0,85 & 1,72 & 1,44 \\
\hline & SB & 0,62 & 0,88 & 0,59 & 0,68 & 0,85 & 0,54 \\
\hline
\end{tabular}

Im pH1.1 (Tab. 11) ist die Streubreite der reinerbig MHS-negativen Endprodukte am geringsten $(0,12-0,16)$, die der Nachkommen der Vaterlinie PI(PP) am höchsten $(0,28)$.

Im pH1.2 dagegen streuen die Nachkommen der Vaterlinie PI(PP) am geringsten $(0,17)$ und die DU(NN)-Kreuzungsendprodukte mit 0,29 am stärksten.

Die höchste Streuung ihrer LF24-Werte zeigen die PI(PP)-Nachkommen mit 1,52, die geringste die Endprodukte der Vaterlinie PI ${ }^{*} \mathrm{HA}(\mathrm{NN})$.

Über alle pH1.1-, pH1.2- und LF24-Messungen hinweg zeigt die Vaterlinie PI*HA(NN) die geringste Variabilität ihrer Kreuzungsendprodukte.

In den Gehaltsmessungen des prozentualen Protein- und Wassergehaltes spiegelt sich der Einfluss des Hampshireeffektes wider. Die höchste Variabilität im Protein- und Wassergehalt kann für die Hampshireherkünfte (Protein (\%): 1,26 - 1,68; Wasser (\%): $0,64-1,15)$ berechnet werden.

Für den intramuskulären Fettgehalt werden hohe Variabilitäten in den Vaterlinien 
(DU(NN) $(0,85 \%)$ und PI(PP) $(0,88 \%)$ berechnet, wobei zu beachten ist, dass das Vaterlinienmittel für die PI(PP)-Nachkommen bei $1,23 \%$, das der DU(NN)-Nachkommen bei $2,03 \%$ liegt (Tab. 8 ).

4.

\section{Diskussion und Schlussfolgerungen}

Ziel des Gesamtversuches (GLODEK, 1999) war es, Möglichkeiten zur Qualitätsverbesserung von Markenschweinefleisch zu eruieren und deren Auswirkungen auf die Gesamtwirtschaftlichkeit der Schweineproduktion (PAULUS, 1999; GLODEK, 1999) zu bestimmen. Angesichts der nahezu weltweiten Uniformität der führenden Hybridsauen-Kombinationen (GLODEK, 1996), wurde als Ansatz der Vergleich der Endprodukte von sechs in Europa verbreiteten Vaterrassen mit deutlich verschiedenen Qualitätsschwerpunkten gewählt. In dieser Teilstudie geht es ausschließlich um die wesentlichen Fleischbeschaffenheitsmerkmale (LAUBE, 2000), die nachfolgend in den praxisüblichen Merkmalskategorien diskutiert werden.

\subsection{PSE und DFD}

Die physiologisch-pathologischen Grundlagen der Defektmutante im Ryanodinrezeptor beschrieben MARTENS $(1997,98)$, ihre molekulare Diagnostik mit dem MHSTest BRENIG und BREM (1992). Erste Rassenvergleiche wurden von FEWSON et al. (1993) publiziert und SCHMITTEN (1993) zeigte, dass auch mischerbige Träger des vorher als rezessiv bewerteten Schadgens deutliche Fleischbeschaffenheitsmängel im PSE-Bereich aufweisen, die von FIEDLER et al. (1993) hinsichtlich der Muskelstruktur bestätigt wurden.

In dieser Feldstudie konnte mit Hilfe des pH-Wertes 85 Minuten p.m. und des LFWertes 24 Stunden p.m. eine klare PSE-Grenze gefunden werden, die auch mischerbige Endprodukte eindeutig identifiziert. Dies war mit dem Reflexions- und pH-Wert 25 Minuten p.m. nicht zuverlässig möglich. Das wesentliche Ergebnis war, dass Nachkommen von MHS-positiven PI(PP)-Vătern etwa 20 \% PSE-gefährdete Schlachtkörper bringen und damit für Markenschweinefleisch nicht in Frage kommen. Auch die heute üblichen Markenschweine von mischerbigen PI*HA(NP)-Vätern lassen noch 10 \% PSE erwarten, was für gehobene Qualităts- und Uniformitătsanspriliche nicht mehr tolerierbar ist (Tab. 6). Dagegen zeigten alle reinerbig stressstabilen Väter, gleichgültig ob PI, PI*HA, DU oder HA*DU, Nachkommen mit unter $2 \%$ PSE-Verdacht und geringer Variabilităt der Indikatormerkmale, womit die theoretischen Erwartungen und fruhere Untersuchungen bestătigt werden. Reinerbig stressresistente Vaterrassen lassen sich mithilfe des MHS-Gentests in kürzester Zeit züchten, was heute allenthalben sogar bei PI-Züchtern geschieht.

Die DFD-Frequenz, gemessen am PH-Wert 24 Stunden p.m., lag bei fünf Endprodukten unter 1,2\% und damit im unproblematischen Bereich (Tab. 7). Die DU-Nachkommen zeigten jedoch fast $3 \%$ der Schlachtkörper uber dieser Grenze, was in einem Exaktversuch (KRATZ, 2000) weiter untersucht wird.

\subsection{Hampshire-Effekt}

In früheren Untersuchungen diente das glykolytische Potenzial im Muskel als Kriterium für diesen dominant vererbten Qualitätsdefekt (LARZUL et al., 1996; LEBRET et al., 1999; FEDDERN et al., 1994), der bisher nur bei Schweinen mit Hampshireanteil gefunden wurde (SURMANN, 1991; WASSMUTH, 1991). Die bei weitem bedeu- 
tendste Komponente des glykolytischen Potenzials ist der Glykogengehalt, der hier als Kriterium benutzt wurde, da mit einem Grenzwert von $10 \mu \mathrm{mol} / \mathrm{g}$ Muskel klar die Tiere mit (> $10 \mu \mathrm{mol} / \mathrm{g}$ ) und ohne Hampshireeffekt getrennt werden konnten. Zwischen diesen beiden Glykogenklassen wurden die aus der Literatur bekannten deutlichen Unterschiede im pH24, dem erhöhten Wasser- und verminderten Proteingehalt des Fleisches gefunden (Tab. 9) und diese bestimmen vor allem die Unterschiede zwischen den Endprodukten mit und ohne HA-Anteil (Tab. 8). Betrachtet man allerdings die Frequenz der Glykogenklassen innerhalb ausreichend großer Nachkommenschaften von HA-Kreuzungsebern (Tab. 10), so zeigt sich, dass von vier der 28 Eber das $\mathrm{RN}^{-}$-Gen nicht vererbt wurde, sie es also von ihren HA-Eltern nicht erhalten hatten. Diese Anteile lassen den Schluss zu, dass in den beteiligten HA-Reinzuchtpopulationen das $\mathrm{RN}^{-}$-Schadgen noch nicht fixiert ist, sondern eine Frequenz von 85-90\% aufweist. Inzwischen ist als Ursache eine Mutation im PRKAG3-Gen gefunden (R200Q), welche über eine muskelspezifische AMPK den Glucosestoffwechsel stört und so den Hampshire-Effekt auslöst (MILAN et al., 2000). Sobald eine Gensonde für diese Mutation verfügbar ist, könnten die HA-Populationen von dem Schadgen befreit werden, wobei allerdings negative Auswirkungen auf andere Merkmale (z.B. den Fleischanteil und die Zunahme) erwartet werden, die noch näher quantifiziert werden müssen.

\subsection{Intramuskulärer Fettgehalt (IMF)}

Die Bedeutung des IMF für die Genussqualität des Fleisches ist in zahlreichen Untersuchungen nachgewiesen worden (KIRCHHEIM et al., 1996; STEINBERG et al., 1996; EIKELENBOOM et al., 1996) und Rassenuntersuchungen liegen von KALLWEIT et al. (1996) in Deutschland vor. Allgemein wird ein Grenzwert von 2-2,5 \% IMF im Longissimus für guten Fleischgeschmack gefordert, den das heutige deutsche Marktangebot bei weitem nicht erreicht. Unter den hier untersuchten sechs Endprodukten (Tab. 8) erreichten nur die DU-Nachkommen mit einem Mittel von $2 \%$ IMF und $40 \%$ der Tiere über dieser Grenze annähernd die erwünschte Qualität, gefolgt von den HA*DU-Nachkommen mit 1,75\% im Mittel und $21 \%$ der Tiere über $2 \%$. Von den vier deutschen Herkünften erreichten die PI*HA(NN)-Nachkommen knapp 1,5\% IMF mit $16 \%$ der Tiere über $2 \%$, die drei anderen lagen unter 1,3\% IMF mit nur 4-6 $\%$ Tiere über $2 \%$ (GLODEK, 1999). Eine Selektion auf erhöhten IMF, wie sie in der Schweiz seit Jahren erfolgreich betrieben wird (SCHWÖRER et al., 1996), dürfte in Deutschland sehr langwierig werden (BRANDT, 1996). Eine schnelle Erhöhung des IMF der Endprodukte ist derzeit nur mit darauf selektierten DU-Vätern aus Dänemark zu erreichen, wobei allerdings auf Grund schlechterer Schlachtkörperqualitäten deutliche Erlöseinbußen bei deutschen Bezahlungsmasken zu erwarten sind (GLODEK, 1999; PAULUS et al., 2000).

\subsection{Ausgeglichenheit der Endprodukte}

Trotz der durchaus repräsentativen Feldbedingungen des Versuches konnte dieser besonders für den Markterlös sehr wichtige Aspekt nicht vollständig untersucht werden, weil die Stichprobengröße mit etwa 10 Besamungsebern (die möglicherweise von den Züchtern unterschiedlich stark selektiert waren) zwar das mittlere Besamungseberangebot, aber wohl kaum die wahre Streuung der jeweiligen Vaterrassen wiedergeben 


\section{konnten.}

Es wurde daher versucht, anhand der Streubreite zwischen Ebernachkommenschaften innerhalb Vaterrassen Unterschiede zwischen den Herkünften sichtbar zu machen (Tab. 11). Dabei zeigte sich erwartungsgemäß, dass nicht reinerbig stressresistente Endproduktkombinationen deutlich die höchsten Streubreiten in den PSE-Indikatoren (pH1.1 und LF24 bei PI(PP)- und pH1.2 und LF24 bei PI*HA(NP)-Ebern) aufwiesen. Für Indikatoren des Hampshirefaktors zeigten Endproduktkombinationen ohne HAAnteile deutlich niedrigere Streubreiten als HA-Kreuzungen, wobei lediglich die 8 PI*HA(NP)-Eber (die alle das $\mathrm{RN}^{-}$-Gen vererbten) recht ausgeglichen waren.

Im intramuskulären Fettgehalt zeigten die fleischreichsten (PI(PP)) und die fettesten (DU) Endprodukte deutlich die höchsten Streubreiten zwischen Vätern und die offenbar am stärksten auf dieses Merkmal selektierten HA*DU-Eber mit nur $0.54 \%$ die geringste. Die besten Selektionsaussichten fur den IMF sind daher in der DU-Rasse gegeben, welche alle anderen hier geprüften Vaterrassen weit hinter sich lässt.

\section{BRANDT, $\mathrm{H}$.:}

\section{Literatur}

Möglichkeiten der Zucht auf höheren intramuskulären Fettgehalt unter deutschen Marktverhăltnissen. IMF-Kolloquium der Thuringer Landesanstalt fur Landwirtschaft Jena (Willhelmsthal), 1996

BRENIG, B.; BREM, G.:

Molecular cloning and analysis of the porcine "halothane" gene. Arch. Tierz., Dummerstorf 35 (1992)1/2, 129-135

EIKELENBOOM, G.; HOVING-BOLINK, A.H.; VAN DER WAL, P.G.: Die Verzehrsqualităt von Schweinefleisch; 2. Einfluss des intramuskulären Fettes. Fleischwirtschaft, Frankfurt/M. 76 (1996) 5, 559-560

FEDDERN, E.; KRIETER, J.; KALM, E.:

Verlauf der postmortalen Glykogenolyse und Merkmale der Fleischbeschaffenheit bei Hampshire-Reinzuchttieren und verschiedenen Kreuzungskombinationen. Arch. Tierz., Dummerstorf 37 (1994), 229243

FEWSON, D.; RATHFELDER, A.; MÜLLER, E.:

Untersuchung uber Beziehung von Fleischanteil, Fleischbeschaffenheit und Stressresistenz bei verschiedenen Schweineherkünften, I. Mitt. Zuchtungskunde, Stuttgart 65 (1993) 4, 284-296

FIEDLER, I.; ENDER, K.; WICKE, M.; LENGERKEN, G. v.:

Zusammenhănge zwischen der Mikrostruktur des Muskelgewebes bei Schweinen der Landrasse und ihrer Stressempfindlichkeit (Halothanreaktion). Arch. Tierz., Dummerstorf 36 (1993), 525-538

GLODEK, P.:

Die Wahl der Vaterlinie bestimmt die Qualităt der Endprodukte in der Schweinezucht. Züchtungskunde, Stuttgart 68 (1996) 6, 483-492

GLODEK, P.:

Die Eignung von Schweinekreuzungen mit besonderen Qualitătseigenschaften zur Verbesserung des deutschen Markenschweineangebotes. Abschlußbericht eines vom Niedersăchsischen Ministerium für Ernăhrung, Landwirtschaft und Forsten, dem VZF-Verbund Uelzen, der Premium AG Zeven und der Züchtungszentrale Deutsches Hybridschwein Lüneburg geforderten gemeinsamen Forschungsvor-habens des Instituts für Tierzucht und Haustiergenetik der Universität Göttingen und der FAL-Institute fur Tierzucht und Tierverhalten, Mariensee und für Tierernăhrung, Braunschweig. pp. 63, 1999

HOFER, A.; SCHWÖRER, D.:

Wie wird der intramuskuläre Fettgehalt honoriert und zilchterisch verbessert? 4. Schweineworkshop Uelzen, pp. 207-216, 1996

KALLWEIT, E.; HENNING, M.; KÖLER, P.; BAULEIN, U.:

Intramuskulärer Fettgehalt verschiedener Schweinerassen. IMF-Kolloquium der Thüringer Landesanstalt fur Landwirtschaft Jena (Willhelmsthal), 1996

KIRCHHEIM, U.; SCHÖNE, F.; REICHARDT, W.; GREILING, A.:

Einfluss des intramuskulăren Fettes auf Parameter der Fleischbeschaffenheit. IMF-Kolloquium der Thüringer Landesanstalt fur Landwirtschaft Jena (Willhelmsthal), 1996 
KRATZ, R.:

Persønliche Mitteilung, 2000

LARZUL, C.; LE ROY, P.; MONIN, G.; SELLIER, P.:

Variabilité génetique du potential glycolytique du muscle chez le porc. INRA Prod. Anim. 11 (1998), 183-197

LAUBE, S.:

Die Eignung spezieller Schweinekreuzungen zur Qualitătsverbesserung von Markenschweinefleisch unter besonderer Berucksichtigung von MHS-Status, Hampshirefaktor und intramuskulärem Fettgehalt. TIHO Hannover, Diss., 2000

LEBRET, B.; LEROY, P.; MONIN, G.; LEFAUCHER, L.; CARITEZ, J.D.; A. TALMANT, A.; ELSEN, J.M.; SELLIER, P.:

Influence of the three RN genotypes on chemical composition, enzym activities, and myofiber charakteristics of porcine skeletal muscle. J. Anim. Sci. 77 (1999), 1482-1489

MARTENS, H.: Physiologie und Pathophysiologie des Ryanodin-Rezeptors beim Schwein. Tierărztliche Praxis 25 (1997), 41-51

MARTENS, H.:

Physiologie der Muskulatur und das MHS-Gen des Schweines: Zur Diskussion um eine Eliminierung des mutierten Ryanodin-Rezeptors aus der deutschen Schweinezucht. Arch. Tierz., Dummerstorf 41 (1998) $1 / 2,179-192$

MILAN, D. et al. :

A mutation in PRKAG 3 associated with excess glycogen content in pig sceletal muscle. Science 288 (2000), 1248-1251

PAULUS, E.-D.:

Die Fleischleistung und der Markterlös von Schweinekreuzungen mit besonderen Qualitätseigenschaften im Vergleich zum heutigen Standard- und Markenschweineangebot. Univ. Grttingen, Diss., 1999

PAULUS, E.D.; HENNING, M.; BRANDT, H.; KALLWEIT, E.; GLODEK, P.:

Die Fleischleistung und der Markterlos von Schweinekreuzungen mit besonderen Qualitătseigenschaften im Vergleich zum heutigen Standard- und Markenschweineangebot. Zuchtungskunde 72 (2000) 4, 290-307

SCHMITTEN, F.:

Schweinefleischqualităt in der Zuchtpraxis. Zuchtungskunde, Stuttgart 65 (1993) 6, 455-467

SCHWÖRER, D.; LORENZ, D.; HOFER, A.; REBSAMEN, A.: Erfolgreiche Steigerung des Genusswertes bei Schweizer Schweinefleisch. Der Kleinviehzllchter 44 (1996), 1095-1130

STEINBERG, M.; BERGFELD, U.; SCHÖBERLEIN, L.:

Untersuchung zum Einfluss des IMF-Gehaltes auf die physikalisch-technologischen Parameter der Fleischbeschaffenheit und die sensorischen Eigenschaften von Schweinefleisch. IMF-Kolloquium der Thuringer Landesanstalt fur Landwirtschaft Jena (Willhelmsthal), 1996

SURMANN, H.:

Vergleich verschiedener Vaterrassen mit und ohne Hampshireanteil im Hinblick auf die Fleischbeschaffenheit und Gesamtwirtschaftlichkeit ihrer Mastendprodukte. Univ. Gottingen, Diss., 1991

WASSMUTH, R.:

Untersuchung zum „Hampshirefaktor" in der Fleischbeschaffenheit von Kreuzungsschweinen mit verschiedenen Anteilen dieser Rasse. Univ. Gøttingen; Diss., 1991

Eingegangen: 01.08 .2000

Akzeptiert: 21.08.2000

Anschriften der Verfasser

Dr. SIGURD LAUBE, Dr. HORST BRANDT,

Prof. Dr. PETER GLODEK

Institut fur Tierzucht $u$. Haustiergenetik

der Universităt Göttingen

Albrecht-Thaer-Weg 3

D-37075 Gorttingen

Dr. MARTINA HENNING,

Prof. Dr. ERHARD KALLWEIT

Institut $\mathrm{f}$. Tierzucht $\mathrm{u}$.

Tierverhalten der FAL

Mariensee

Holtystr. 10

D-31535 Neustadt 